\title{
Review Article \\ Beam Purity for Light Dark Matter Search in Beam Dump Experiments
}

\author{
D. Banerjee, P. Crivelli, and A. Rubbia \\ Institute for Particle Physics, ETH Zurich, 8093 Zurich, Switzerland \\ Correspondence should be addressed to P. Crivelli; paolo.crivelli@cern.ch
}

Received 23 March 2015; Accepted 16 June 2015

Academic Editor: Torsten Asselmeyer-Maluga

Copyright (C) 2015 D. Banerjee et al. This is an open access article distributed under the Creative Commons Attribution License, which permits unrestricted use, distribution, and reproduction in any medium, provided the original work is properly cited. The publication of this article was funded by $\mathrm{SCOAP}^{3}$.

\begin{abstract}
This paper reviews the search for light dark matter in beam dump experiments with a special emphasis on the necessity of beam purity for precise background rejection at the sensitivities aimed at these experiments. As a case study we cite the P348 experiment which has test beam time in Fall 2015 at the SPS H4 beam line at CERN and aims to search for the $U^{\prime}(1)$ gauge boson, $A^{\prime}$, which as per one model of dark matter mediates a weak interaction between ordinary matter and dark matter via mixing of these "dark photons" with ordinary photon. The experiment aims to probe the still unexplored area of mixing strength $10^{-5} \leq \epsilon \leq 10^{-3}$ and masses $M_{A^{\prime}} \leq 100 \mathrm{MeV}$ by using $10-300 \mathrm{GeV}$ electron beam from the CERN SPS. This paper presents the simulation results for rejection of background due to beam impurity, by tracking the incoming particles with Micromegas detectors at a level $<10^{-10}$.
\end{abstract}

\section{Introduction}

The existence of dark matter has been postulated primarily because of the observation that the motion of galaxies within a cluster suggests they are bound by a total gravitational force due to about 5-10 times as much matter as can be accounted for from luminous matter in the said galaxies. It is possible to measure the rate of rotation of the stars about the galactic centre. The resultant "rotation curve," related to the distribution of matter in the galaxy, suggests the outer stars seem to rotate too fast for the amount of observable matter in the galaxy. These results can be explained assuming there is a "dark matter halo" surrounding every galaxy which unlike normal matter does not interact via the electromagnetic force making it extremely hard to detect. Further observations and theories supporting the existence of dark matter are reviewed in [1-3]. Various experiments are underway which aim to search for dark matter in the $\mathrm{GeV}-\mathrm{TeV}$ mass range, the socalled WIMPs (most popular candidate for dark matter), but these experiments are essentially blind to the search for light dark matter in the $\mathrm{MeV}-\mathrm{GeV}$ range. Models of dark matter in our specified mass range $(\mathrm{MeV}-\mathrm{GeV})$ can account for its observed abundance through either thermal freezeout or nonthermal mechanisms [4-8]. Among the better motivated models of $\mathrm{MeV}-\mathrm{GeV}$ dark matter are those whose interactions with ordinary matter are mediated by new "dark" force carriers, namely, a $U^{\prime}(1)$ gauge boson, $A^{\prime}$ (dark photon) that kinetically mixes with ordinary photon as in $[9,10]$

$$
L_{\text {int }}=-\frac{1}{2} \epsilon F_{\mu \nu} A^{\prime \mu \nu},
$$

where $F_{\mu \nu}, A^{\prime \mu \nu}$ are the ordinary and dark photon fields, respectively, and parameter $\epsilon$ is their mixing strength. In a class of models, $A^{\prime}$ may have a mass $M_{A^{\prime}} \leq 100 \mathrm{MeV}$ and $\gamma-A^{\prime}$ mixing strength as large as $\epsilon \sim 10^{-5}-10^{-3}$, which is in the experimentally accessible and theoretically interesting region [11, 12]. Dark matter in this mass range can be probed in beam dump experiments or through rare particle decay; for example, if the $A^{\prime}$ mass is below the mass of $\pi^{0}$ it can be searched for in the decay of $\pi^{0} \rightarrow \gamma A^{\prime}$ and the subsequent decay of $A^{\prime}$ to $e^{+} e^{-}$pairs [13-16]. Recently stringent constraints on the mixing parameter $\epsilon$ in the sub$\mathrm{GeV}$ mass range have been derived from a search of this decay mode with existing data of neutrino experiments $[17,18]$ and from SN1987A cooling [19]. This makes further searches for dark mediators interesting. 


\section{Principle of Detection}

The experimental principle of these searches includes having a detector downstream of a high energy electron beam to detect these "dark" force carriers from their SM decay products, quasi-elastic nucleon scattering or detecting missing energy downstream of the beam from their invisible decay products. In this paper we will focus mainly on the invisible decay channel. A high-intensity, high energy electron beam impinges on a beam dump target producing dark sector states which are detected downstream with various detectors and vetoes.

The visible decay channel, that is, $A^{\prime} \rightarrow e^{+} e^{-}$, can be searched for in a "light-shining-through-a-wall-experiment" wherein $A^{\prime}$ does not interact with any component of the detector geometry but $e^{+} e^{-}$are tracked by trackers placed downstream which does not appear to originate from any primary particle, like a light shining through a wall. The invisible decay channel can be searched for by detecting missing energy downstream. Such an approach has been taken in the P348 experiment due in CERN with a beam time in Fall 2015 at the SPS H4 beam line.

For the invisible decay channel when $A^{\prime}$ 's produced decay into dark matter particle pairs, the decay products do not interact with any detector thereafter. Thus, the total of the incoming beam energy will not be accounted for in the total deposited energy downstream of the target. For such signatures, it is very important to ensure there is no leakage of energy to minimise the possibility of false signal and also the incoming energy should be well known to accurately calculate the percent of missing energy. These searches are limited mainly due to leakage of energy, beam impurity (contamination from hadrons) with the hadrons decaying to neutrinos, and a lepton before the detector with the neutrino carrying most of the energy undetected and due to a broad energy spectrum of the primary beam. For the first two cases it is important to ensure complete hermeticity of the detector to minimise leakage and proper primary particle identification to reject hadrons. Since these experiments require sensitive searches it is absolutely crucial to estimate the energy of the incoming particles to accurately reject background from possible low energy tail of the primary beam mimicking a positive signal. As a case study we will present the P348 experiment which aims to use Micromegas detectors to accurately track these incoming particles in a magnetic field to estimate their energy.

\section{P348 Experiment}

The P348 experiment aims to search for the dark force carriers mentioned above and probe the still unexplored area of mixing strength $10^{-5} \leq \epsilon \leq 10^{-3}$ and masses $M_{A^{\prime}} \leq$ $100 \mathrm{MeV}$ using the $10-300 \mathrm{GeV}$ electron beam present at the CERN SPS. $A^{\prime \prime}$ s with such parameters are short-lived and decay with a lifetime $<10^{-10} \mathrm{~s}$ into their SM $e^{+} e^{-}$pairs or dark particles. Figure 1 shows the exclusion plots expected from the $A^{\prime} \rightarrow$ invisible channel search and also the already existing constraints obtained from previous experiments. The light blue and blue area show the expected 90\% C.L.

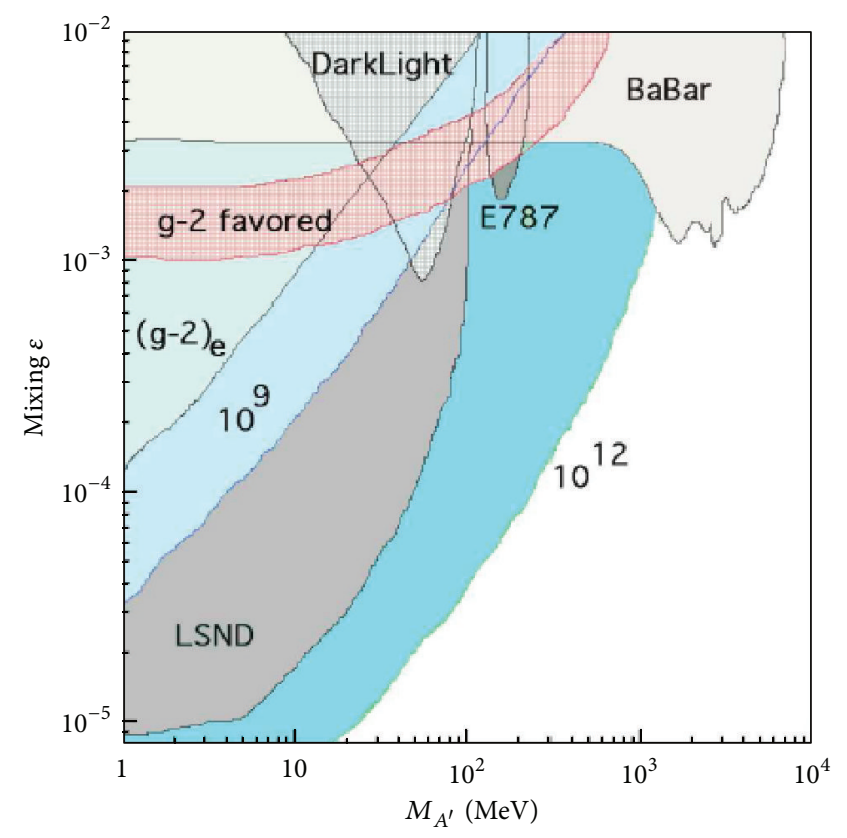

FIgURE 1: Exclusion region in $\left(M_{A^{\prime}} ; \epsilon\right)$ plane for invisibly decaying $A^{\prime}$ into a pair of light dark matter particles $\chi \bar{\chi}$, provided $M_{A^{\prime}}>2 m_{\chi}$.

exclusion areas corresponding, respectively, to $10^{9}$ and $10^{12}$ accumulated electrons at $100 \mathrm{GeV}$ for the background-free case. Other existing constraints mostly adapted from [20] are also shown. The constraint from the BaBar monophoton search is given as the light grey shaded region. Further limits are shown from the anomalous magnetic moment of the electron $\left((g-2)_{e}\right)$ and DarkLight, the rare kaon decay $K^{+} \rightarrow \pi^{+} A^{\prime}$ (E787), and LSD experiments. The LSND area is determined assuming $A^{\prime}-\chi$ coupling $\alpha_{D}=0.1$, and that $\chi$ cannot decay to other light dark sector states which do not interact with $A^{\prime \prime}$ s [21]. The red shaded region is preferred in order to explain the discrepancy between the measured and the predicted value of the anomalous magnetic moment of the muon.

3.1. Experimental Setup for P348. The P348 experiment has the same basic experimental setup for both $A^{\prime} \rightarrow e^{+} e^{-}$and $A^{\prime} \rightarrow$ invisible searches as shown in Figure 2 [22] with ECAL1 and ECAL2 for detecting electromagnetic showers, a decay volume (DV) for the decay of $A^{\prime}$, tracking detectors, DHRMM1 and DHRMM2, for tracking the $e^{+} e^{-}$tracks, and a HCAL mainly for the invisible decay channel and vetoes. The setup before the ECAL1 is to tag the incoming electrons as is explained later.

\section{The Invisible Decay Channel}

The rate of events of $A^{\prime}$ production and its subsequent decay to dark particles in the previously mentioned parameter space is at $\leq 10^{-10}$ with respect to the ordinary photon production rate. In order to be able to reach such statistics and observe a successful $A^{\prime}$ event, the P348 experiment aims to use 


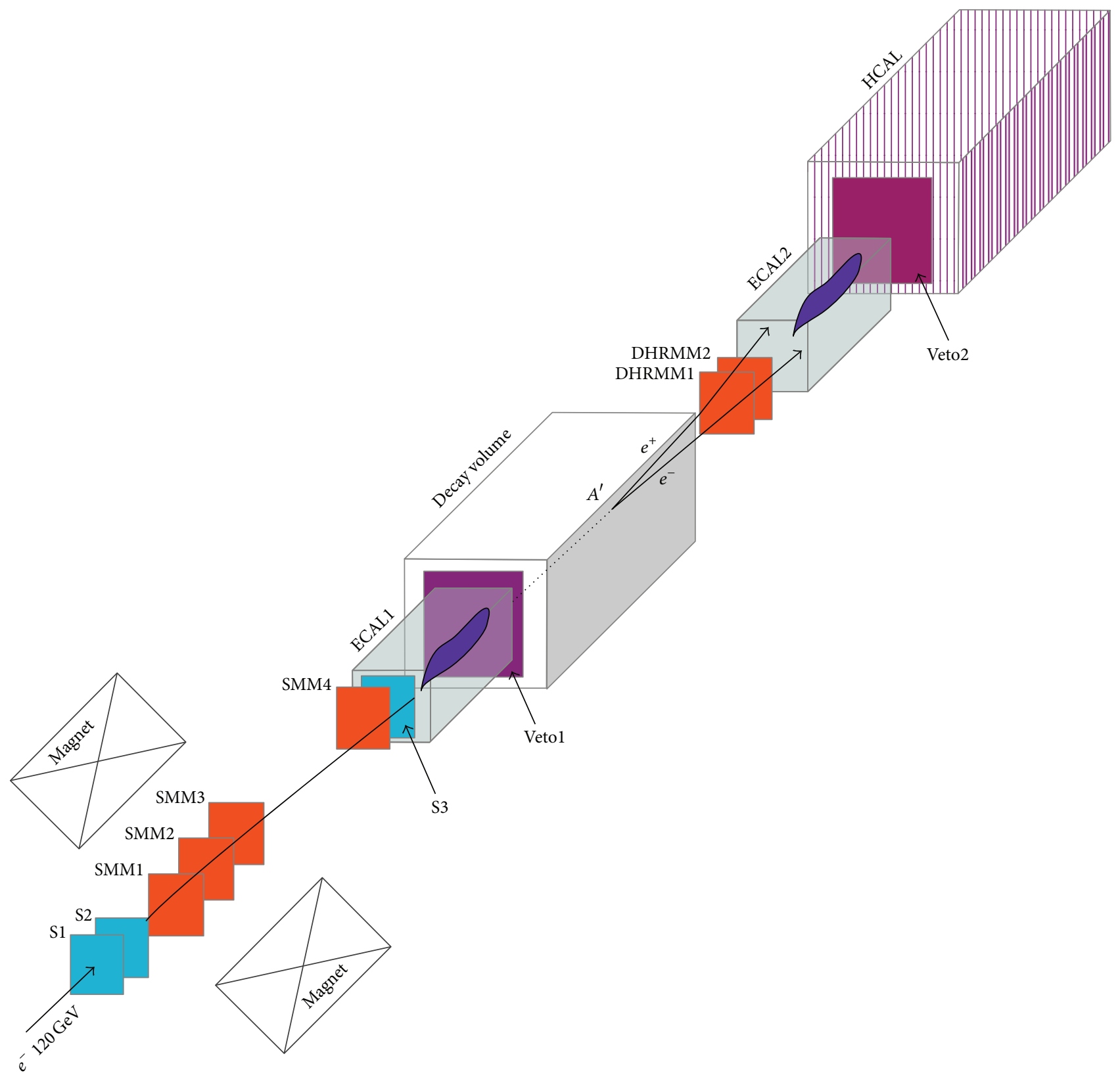

FIGURE 2: Experimental setup for the P348 experiment.

the CERN SPS H4 $e^{-}$beam at an energy $\sim 100 \mathrm{GeV}$ and a maximal beam intensity of $\sim 10^{5} e^{-}$for one typical SPS spill cycle of $14.8 \mathrm{~s}$ including $4.8 \mathrm{~s}$ spill duration and maximum four cycles per minute. The beam impurity from other charged particles in the beam is below $10^{-2}$ and the size of the beam at ECAL1 is of the order of a few $\mathrm{cm}^{2}$. To estimate the exact beam energy spectrum and its low energy tail a full beam line simulation is required at a high level of precision; however, the fraction of electrons with energy $E<E_{\text {th }}$ where $E_{\text {th }}=10 \mathrm{GeV}$ is expected to be $<10^{-6}$. The origin of the low energy tail is mainly due to the interactions of the beam with passive materials, such as entrance windows of the beam lines and residual gas and also due to the in-flight decay of pions and muons in the beam line. The incoming high energy electron beam impinges on the ECAL1 and interacts with the material. $A^{\prime}$ 's are produced through mixing with bremsstrahlung photons from the electrons scattering off the nuclei in ECAL1. A fraction $(f)(10 \%)$ of the beam energy $E_{1}=f E_{0}=10 \mathrm{GeV}$, where $E_{0}$ is the beam energy $=100 \mathrm{GeV}$, is deposited in the ECALl while the remaining $90 \mathrm{GeV}$ is carried by the $A^{\prime}$. It goes undetected through the first veto into the decay volume (DV) where it decays into dark matter particles. The decay products go undetected through S1, S2, veto1, ECAL2, veto2, and the HCAL. So there is a missing $\sim 90 \mathrm{GeV}$ energy signal for this channel in the downstream part of ECAL1. Instead if a low energy electron, $\sim 10 \mathrm{GeV}$, impinges on the ECALl and deposits all its energy there, no signal thereafter in the detectors downstream will mimic our 


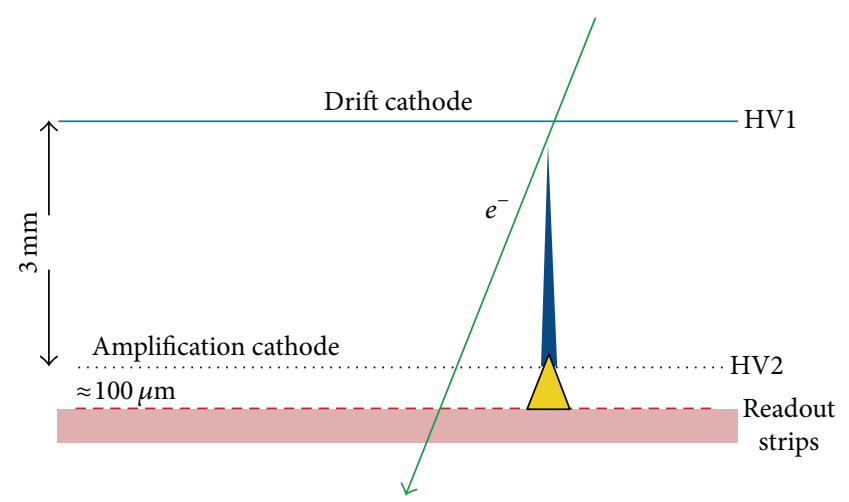

FIgURE 3: Schematic of the principle of operation of a Micromegas detector showing an incoming charged particle ionising the gas and leaving a signal on the readout strips.

event signature and give false signal. It is, therefore, crucial to be completely certain of the energy of the incoming particle to eliminate the background at the level required.

We propose to use a scheme to tag the incoming electron beam by tracking them in a magnetic field using $X-Y$ Micromegas detectors.

\section{Micromegas Detector for Low Energy Background Rejection}

The detector is a two-stage parallel plate avalanche gaschamber, with Ar-isobutane mixture (95-5\%), grounded copper readout strips, and nickel electroformed micromesh for the drift and amplification cathodes. The drift cathode is maintained at $3 \mathrm{~mm}$ from the amplification cathode with a drift voltage of $1 \mathrm{kV} / \mathrm{cm}$ and the amplification cathode is maintained at $128 \mu \mathrm{m}$ from the readout strips as shown in Figure 3 with an amplification voltage of $40 \mathrm{kV} / \mathrm{cm}$. A charged particle entering the detector ionises the gas and produces secondary electrons which drift towards the amplification mesh under the applied E-field. The secondary electrons on reaching the amplification mesh produce avalanche like secondary ionisation due to the high amplification field. The resultant signal thus induced is a sum of the electron and ion signal. The P348 experiment aims to use the $X-Y$ Micromegas design with $250 \mu \mathrm{m}$ strip pitch. The designs of the detector with the corresponding strip widths are shown in Figure 4. The detectors for this experiment will be equipped with a resistive layer as shown to limit the sparks due to the high beam rate. The material budget of the detector is minimised using a "honey comb" structure for the PCB, which includes two $100 \mu \mathrm{m}$ FR4 PCBs with supporting pillars between them in the active area and $3 \mathrm{~mm}$ FR4 PCB outside the active area. This has been designed and produced in the Micromegas workshop of Rui de Oliveira at CERN.

In order to track the incoming particle the detectors will be placed in the yoke of a magnet already present in the $\mathrm{H} 4$ beam area with a field strength of $1.5 \mathrm{~T}$ for a yoke of $17 \mathrm{~cm}$ over a distance of $2 \mathrm{~m}$. As shown in Figure 2 there are two scintillators ( $\mathrm{S} 1$ and S2) placed before the Micromegas

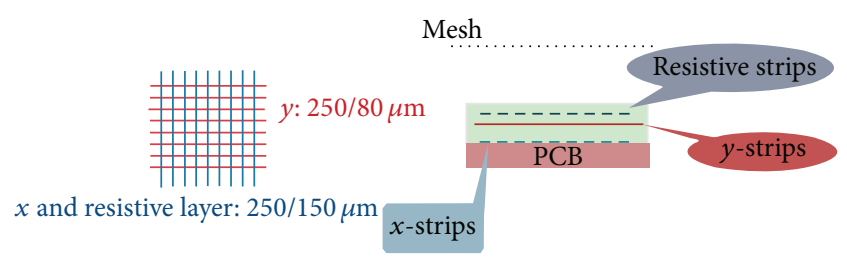

Figure 4: $X-Y$ Micromegas detectors showing the dimensions of the strips and the resistive layer. The resistive layer helps to limit the spark at high rate. The $X-Y$ module helps to determine the hit position of the incoming particle and track it precisely in the magnetic field.

modules (SMM1, SMM2, and SMM3) and the magnet which will be used to monitor the beam and as a trigger for the Micromegas modules. Between each Micromegas in the magnet area will be a vacuum tube to reduce secondary interaction of the primary beam with air. The beam will go through the scintillators and enter the magnet where it will be tracked by the three Micromegas modules. The three Micromegas modules will be placed about $1 \mathrm{~m}$ apart in the $2 \mathrm{~m}$ magnet. The ECAL1 will be placed $15 \mathrm{~m}$ (vacuum tube in between) from SMM3. To get the hit point of the primary beam on the ECAL1 with good spatial resolution a fourth Micromegas module (SMM4) will be placed right before the ECAL1 followed by another scintillator (S3) for good time resolution of the beam entering the ECAL1. The ECAL1 with SMM4 and S3 will be placed offset by $12 \mathrm{~cm}$ in the direction of the deflection to account for the deflection of the electron in the magnetic field.

Simulations have been carried out with Geant 4 to estimate the level of background rejection with this setup. The momentum reconstruction is limited mainly due to the interactions of the primary beam with the material of the detector. The simulations included Mylar windows as the in- and output flanges to the vacuum tubes in each detector module. The beam divergence $\sigma \sim 50 \mu \mathrm{rad}$ and beam diameter $\sigma \sim 0.7 \mathrm{~cm}$ (values obtained from Ilias Efthymiopoulos of CERN SPS) were also taken into account. Due to the limitation of the magnet yoke diameter the dimension of the Micromegas modules that can be placed in the magnet is $=8 \mathrm{~cm} \times 8 \mathrm{~cm}$. Because of the size of the modules, the detector efficiency, the beam diameter, and divergence, simulations show $95 \%$ efficiency for tracking. $10^{6}$ events with $10 \mathrm{GeV}$ and $30 \mathrm{GeV}$ primaries were simulated and the momentum of the track when there is an electron entering the ECAL1 was reconstructed. Due to the offset of the ECAL none of the $10 \mathrm{GeV}$ primaries hit the ECAL $15 \mathrm{~m}$ away even at a level of $10^{-7}$.

Figure 5 shows the spectrum of momentum reconstructed for $30 \mathrm{GeV}$ primary electrons. As seen from the plot out of the $10^{6}$ simulated events $\sim 0.1 \%$ had an actual hit in ECAL1 with majority of the primary being deflected away due to the magnetic field. Of the events when there is some hit in ECAL1 there is no reconstructed momentum $>50 \mathrm{GeV}$ to mimic a false signal. The tail of lower reconstructed momenta is due to the loss of energy of the primary electrons due to interaction in the detector materials. The reconstruction was 


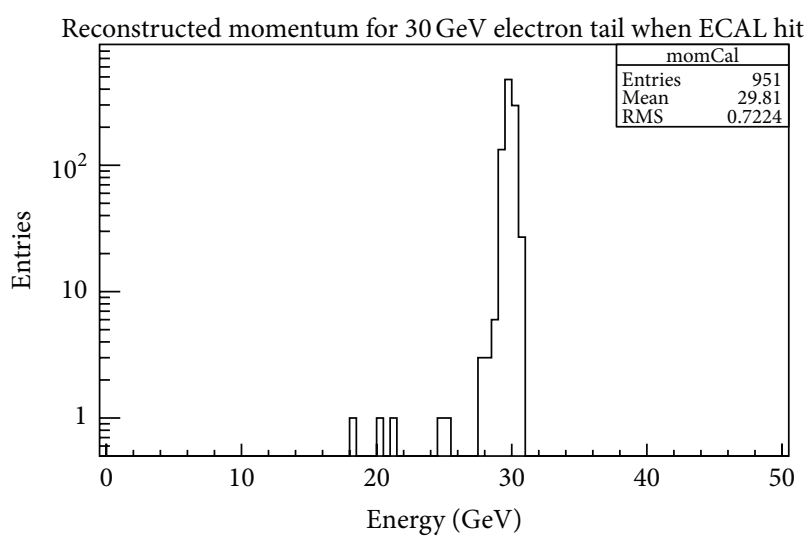

Figure 5: Reconstructed momentum of $30 \mathrm{GeV}$ primaries from Geant4 simulation when there is an actual hit in ECAL1.

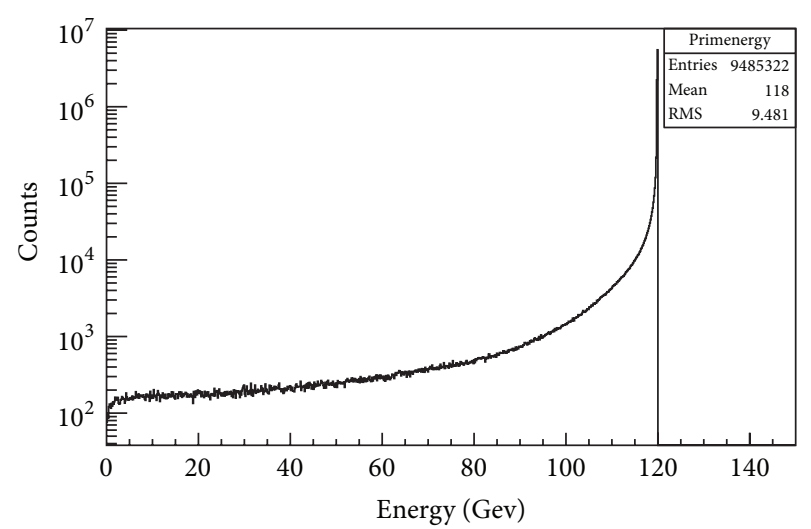

Figure 6: Spectrum of energy of $120 \mathrm{GeV}$ primary beam after interaction with the detector materials before the ECAL1. The inefficiency in the beam with $E<50 \mathrm{GeV}$ is $\sim 0.7 \%$.

also checked for higher statistics, $\sim 10^{7}$ simulated events, and it was noticed that due to the interaction of the primaries with the detector materials and the hit points being given by the secondary tracks instead of the primary there were 7 events with reconstructed momenta $>50 \mathrm{GeV}$ for the $30 \mathrm{GeV}$ primaries. Thus the background can efficiently be rejected by tracking at the level of $10^{-6}$ for the $30 \mathrm{GeV}$ primaries.

As seen from Figure 6, the spectrum of the energy deposited in ECAL1 by $120 \mathrm{GeV}$ primary electrons has a long low energy tail which results in an inefficiency of the experiment and is also a source of background wherein the high energy primary after losing most of its energy hits the ECALl as a low energy primary or in case the high energy primary after interacting with the trackers completely missed the ECAL1 and its low energy secondaries hit the ECAL1 (as shown in Figure 7). It is thus important to make sure that these primaries do not add to any false signal probability when they reach the ECAL1. The inefficiency due to these interactions is $\sim 0.7 \%$. An analysis was done to check at what level this low energy background can be rejected with tracking and the level at which such backgrounds can be effectively suppressed is $<10^{-10}$. This beam spectrometer

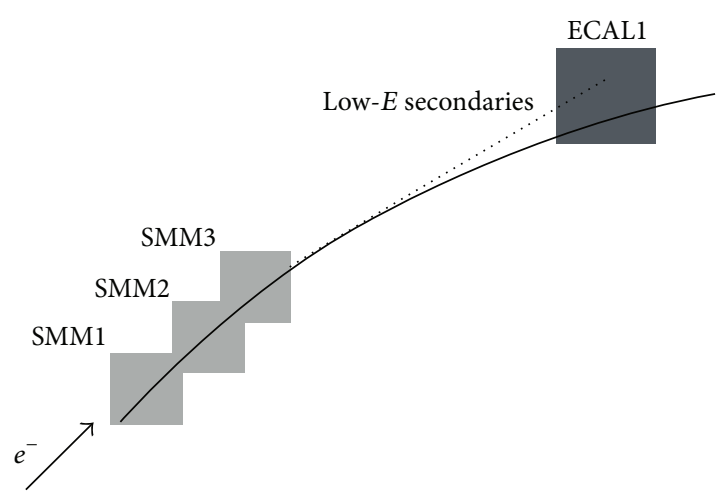

FIGURE 7: Track showing primary high energy missing the ECAL1 and the low energy secondaries hitting the ECAL1 which can be rejected from the hit point on SMM4 before the ECAL1 and the trajectory of the primary track in the first three Micromegas modules.

system using the Micromegas modules in a magnet thus effectively suppresses the low energy background at the level required and meets the necessity for beam purity as explained previously for detecting missing energy in these experiments.

\section{Conclusion}

Thus we present the importance of beam purity in the searches of dark force mediators by their invisible decay channel, with the P348 experiment as a case study. We present a scheme and preliminary simulation results to use $X-Y$ Micromegas detectors as a mass spectrometer to tag the incoming beam in a magnetic field as will be done in the P348 experiment at CERN. The experiment has test beam time in Fall 2015 wherein the scheme for tracking can be tested and the simulation results can be verified experimentally as well.

\section{Conflict of Interests}

The authors declare that there is no conflict of interests regarding the publication of this paper.

\section{References}

[1] D. N. Spergel and P. J. Steinhardt, "Observational evidence for self-interacting cold dark matter," Physical Review Letters, vol. 84, no. 17, pp. 3760-3763, 2000.

[2] G. Bertone, D. Hooper, and J. Silk, "Particle dark matter: evidence, candidates and constraints," Physics Reports, vol. 405, no. 5-6, pp. 279-390, 2005.

[3] L. Bergström, "Non-baryonic dark matter: observational evidence and detection methods," Reports on Progress in Physics, vol. 63, no. 5, pp. 793-841, 2000.

[4] D. B. Kaplan, "Single explanation for both baryon and dark matter densities," Physical Review Letters, vol. 68, no. 6, pp. 741743, 1992.

[5] M. Pospelov and A. Ritz, "Astrophysical signatures of secluded dark matter," Physics Letters B, vol. 671, no. 3, pp. 391-397, 2009.

[6] J. L. Feng, "Non-WIMP candidates," in Particle Dark Matter: Observations, Models and Searches, G. Bertone, 
Ed., chapter 10, pp. 190-204, Cambridge University Press, 2010, http://www.cambridge.org/ch/academic/subjects/physics/cosmology-relativity-and-gravitation/particle-dark-matter-observations-models-and-searches? format=HB.

[7] A. Katz and R. Sundrum, "Breaking the dark force," Journal of High Energy Physics, vol. 2009, no. 6, article 003, 2009.

[8] S. Pirandola, A. Serafini, and S. Lloyd, "Correlation matrices of two-mode bosonic systems," Physical Review A, vol. 79, Article ID 052327, 2009.

[9] L. B. Okun, "Limits of electrodynamics: paraphotons?" Zhurnal Eksperimentalnoi i Teoreticheskoi Fiziki, vol. 83, pp. 892-898, 1982.

[10] L. B. Okun, "Limits of electrodynamics: paraphotons?" Soviet Physics-JETP, vol. 56, p. 502, 1982.

[11] J. Jaeckel and A. Ringwald, "The low-energy frontier of particle physics," Annual Review of Nuclear and Particle Science, vol. 60, no. 1, pp. 405-437, 2010.

[12] J. L. Hewett, H. Weerts, R. Brock et al., "Fundamental physics at the intensity frontier," http://arxiv.org/abs/1205.2671.

[13] F. Archilli, D. Babusci, D. Badoni et al., "Search for a vector gauge boson in $\phi$ meson decays with the KLOE detector," Physics Letters B, vol. 706, no. 4-5, pp. 251-255, 2012.

[14] B. Aubert, Y. Karyotakis, J. P. Lees et al., "Search for dimuon decays of a light scalar boson in radiative transitions $\Upsilon \rightarrow \gamma A^{0}$," Physical Review Letters, vol. 103, no. 8, Article ID 081803, 7 pages, 2009.

[15] S. N. Gninenko, "Constraints on dark photons from $\pi^{0}$ decays," Physical Review D, vol. 87, no. 3, Article ID 035030, 4 pages, 2013.

[16] G. Agakishiev, A. Balanda, D. Belver et al., "Searching a dark photon with HADES," Physics Letters B, vol. 731, pp. 265-271, 2014.

[17] S. N. Gninenko and N. V. Krasnikov, "On search for a new light gauge boson from $\pi^{0}(\eta) \rightarrow \gamma+X$ decays in neutrino experiments," Physics Letters B, vol. 427, no. 3-4, pp. 307-313, 1998.

[18] J. Altegoer, P. Astier, D. Autiero et al., "Search for a new gauge boson in $\pi^{0}$ decays," Physics Letters B, vol. 428, pp. 197-205, 1998.

[19] J. B. Dent, F. Ferrer, and L. M. Krauss, "Constraints on light hidden sector gauge bosons from supernova cooling," http://arxiv.org/abs/1201.2683.

[20] R. Essig, J. Mardon, and T. Volansky, "Direct detection of subGeV dark matter," Physical Review D, vol. 85, Article ID 076007, 2012.

[21] P. deNiverville, M. Pospelov, and A. Ritz, "Observing a light dark matter beam with neutrino experiments," Physical Review D, vol. 84, no. 7, Article ID 075020, 2011.

[22] S. N. Gninenko, "Search for $\mathrm{MeV}$ dark photons in a lightshining-through-walls experiment at CERN," Physical Review D, vol. 89, no. 7, Article ID 075008, 10 pages, 2014. 

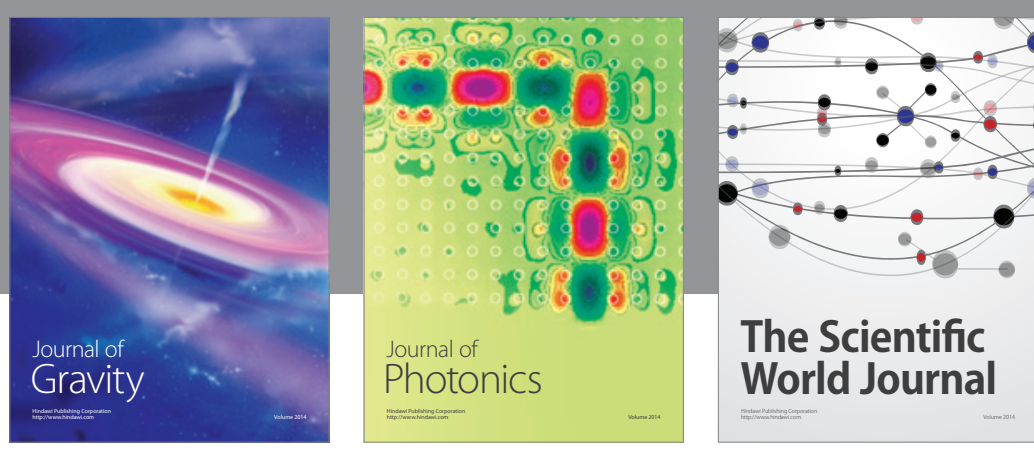

The Scientific World Journal
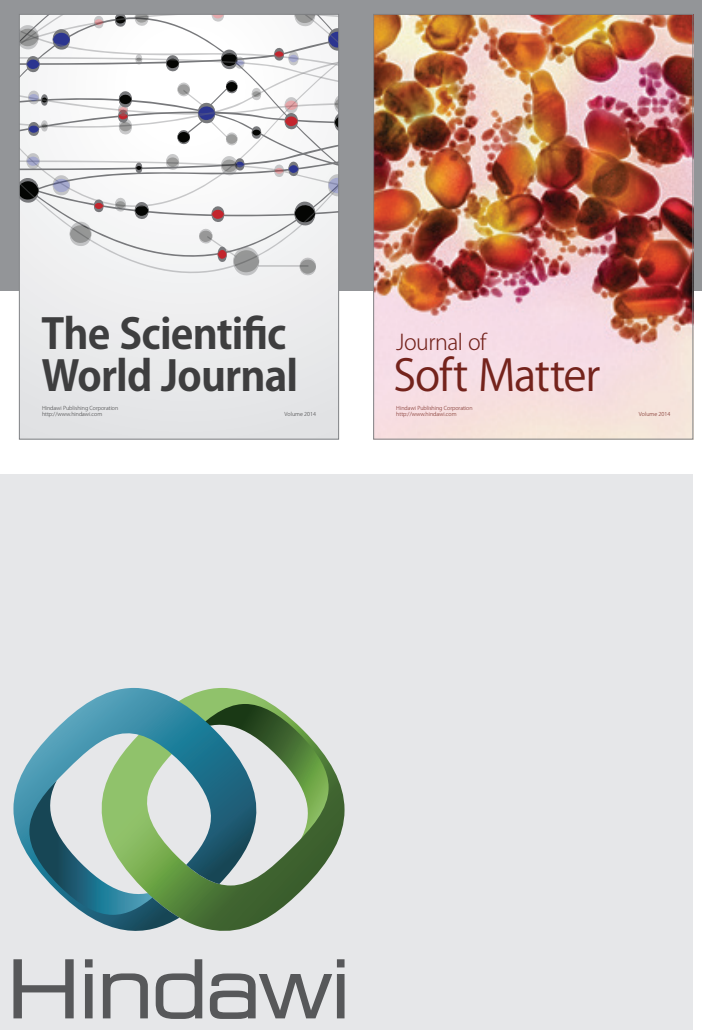

Submit your manuscripts at

http://www.hindawi.com

nternational Journal of

Statistical Mechanics
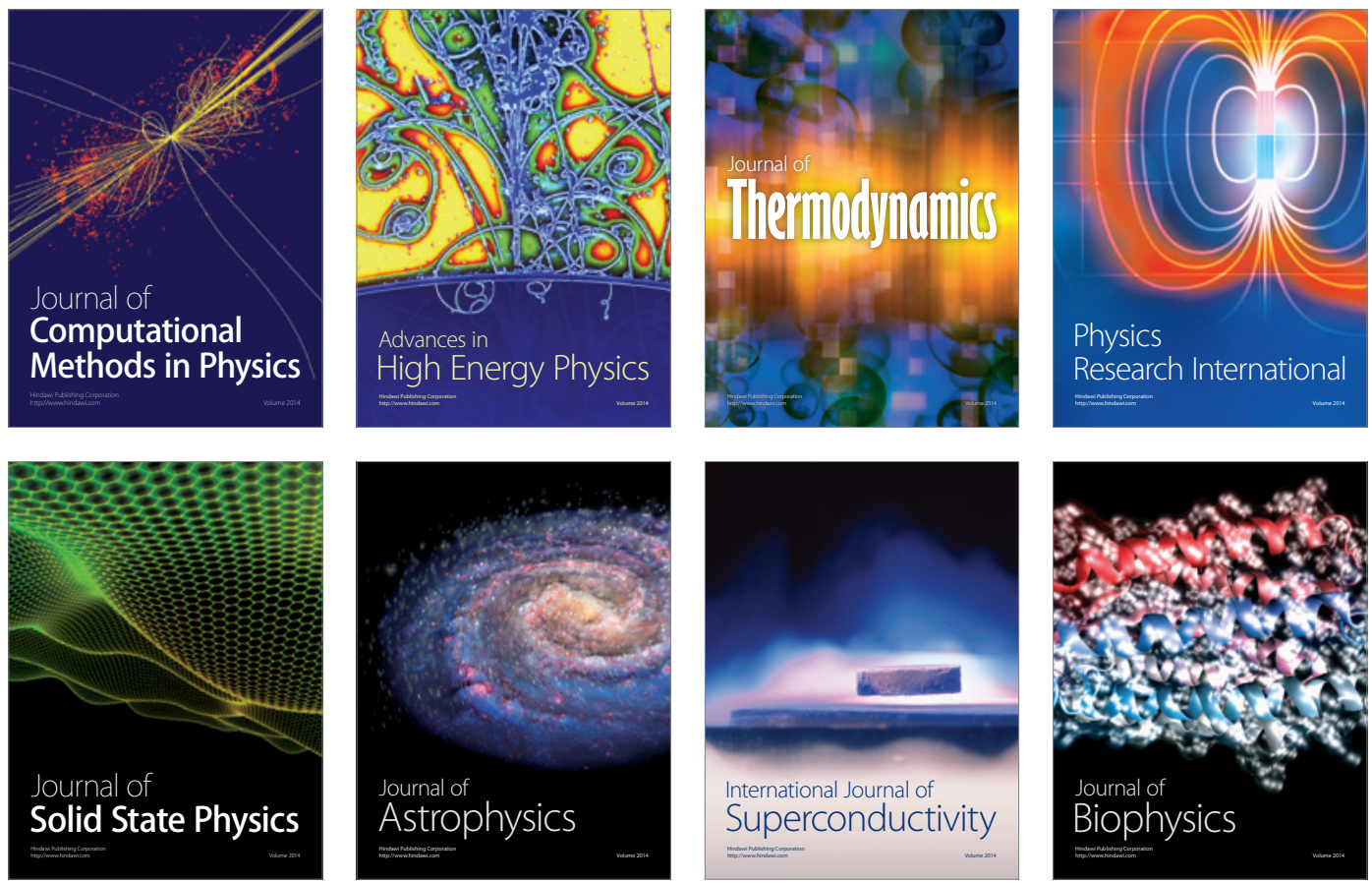
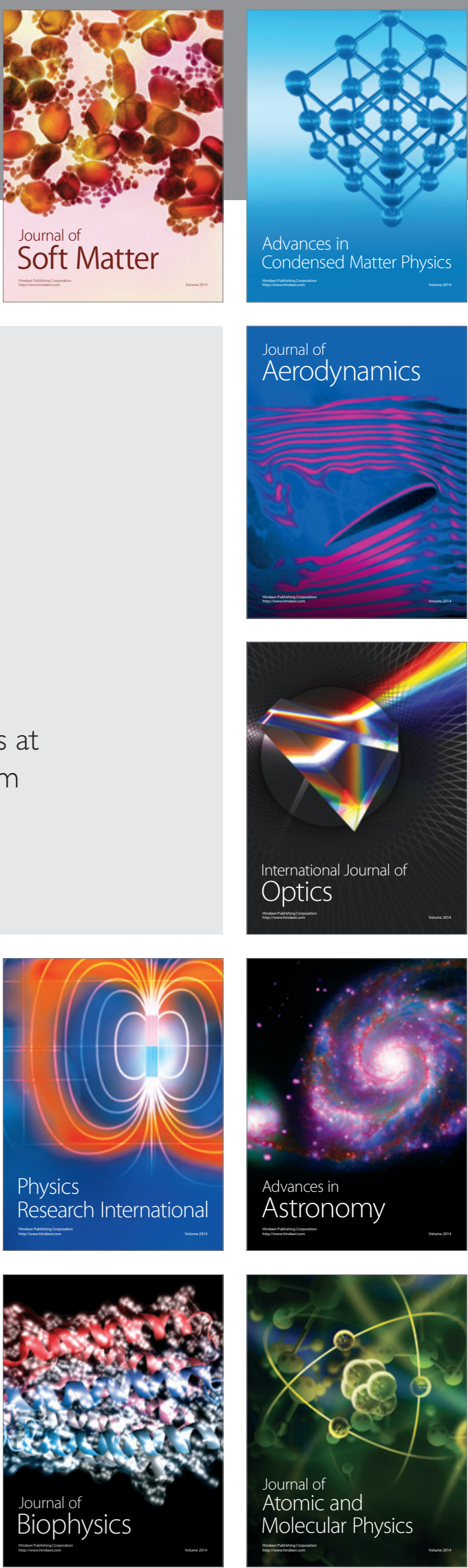